EPJ manuscript No.

(will be inserted by the editor)

\title{
Quasiparticle states in superconducting superlattices
}

\author{
Mihajlo Vanevića and Zoran Radović \\ Department of Physics, University of Belgrade, P. O. Box 368, 11001 Belgrade, Serbia and Montenegro
}

Received: November 21, 2018/ Revised version: date

\begin{abstract}
The energy bands and the global density of states are computed for superconductor / normalmetal superlattices in the clean limit. Dispersion relations are derived for the general case of insulating interfaces, including the mismatch of Fermi velocities and effective band masses. We focus on the influence of finite interface transparency and compare our results with those for transparent superlattices and trilayers. Analogously to the rapid variation on the atomic scale of the energy dispersion with layer thicknesses in transparent superlattices, we find strong oscillations of the almost flat energy bands (transmission resonances) in the case of finite transparency. In small-period transparent superlattices the BCS coherence peak disappears and a similar subgap peak is formed due to the Andreev process. With decreasing interface transparency the characteristic double peak structure in the global density of states develops towards a gapless BCS-like result in the tunnel limit. This effect can be used as a reliable STM probe for interface transparency.

PACS. 74.45.+c Proximity effects; Andreev effect; SN and SNS junctions
\end{abstract}

\section{Introduction}

The artificial $\mathrm{S} / \mathrm{N}$ superlattices consisting of alternating superconductor $(\mathrm{S})$ and normal-metal or semiconductor (N) layers have been studied for some time already 12345 see also 1011. The recent advancement of nanofabrication technology and experimental techniques [12, as well as intrinsically layered structure of high- $T_{c}$ superconductors [13 14 15] has reinvigorated the long standing interest in understanding the effects inherent to clean superconducting heterostructures 161718 . The size and coher, ence effects have been studied recently for double barrier SNS and NSN junctions in the clean limit based on the solutions of Gor'kov and Bogoliubov-de Gennes (BdG) equations 192021222324 .

In this paper we extend the previous approach of Tanaka and Tsukada 6 and Plehn et al. 8 to the more general case of superlattices with finite interlayer transparency. We present comprehensive and systematic analysis of the influence of interface transparency on the quasiparticle band structure and density of states for wide range of the superlattice parameters. Due to the phase coherence of electronic wave functions the energy spectrum is gapless in superlattices with thin $\mathrm{S}$ layers and transparent interfaces 24, and splits into almost flat bands (transmission resonances) with decreasing transparency. For thick S layers, the subgap bands are formed due to the Andreev reflection [25] which leads to the conversion

a Present address: Department of Physics, University of Basel, Klingelbergstrasse 82, 4056 Basel, Switzerland; e-mail: mihajlo.vanevic@unibas.ch of Cooper pairs in superconducting layers into correlated electrons and holes in the normal layers. Whereas the calculations are performed in the clean limit, the influence of impurities on the density of states can be taken into ac6989 by replacing the superconducting coherence length with an effective one, as shown by Halterman and Valls [26] in comparison with experiments of Moussy et al. [12. Our results for density of states in superlattices with layer thicknesses smaller than the superconducting correlation length, qualitatively confirm main features previously obtained by Bulaevskii and Zyskin 4 and Buzdin et al. 5] for atomic-scale layered systems within the tight binding approximation.

\section{The model}

The system under consideration is an $\mathrm{S} / \mathrm{N}$ superlattice in the clean limit, consisting of alternating superconducting and normal-metal (or semiconductor) layers of thickness $d_{S}$ and $d_{N}$, with insulating interfaces modelled as thin potential-energy barriers. The superconducting layers are characterized by constant pair potential $\Delta_{0}$, and zero phase difference, $\phi=0$, is assumed across the superlattice. Effective band masses and electrostatic potentials of the two metals are $m_{S}\left(m_{N}\right)$ and $U_{S}\left(U_{N}\right)$, respectively. The superlattice is uniform in the $x-y$ plane and the $z$ axis is perpendicular to the layers.

Quasiparticle propagation in the superlattice is described by the Bogoliubov-de Gennes equation

$$
\left(\begin{array}{cc}
H_{0}(\mathbf{r}) & \Delta(\mathbf{r}) \\
\Delta^{*}(\mathbf{r}) & -H_{0}^{*}(\mathbf{r})
\end{array}\right) \Psi(\mathbf{r})=E \Psi(\mathbf{r})
$$


where $\Psi(\mathbf{r})=(u(\mathbf{r}), v(\mathbf{r}))^{T}$ is the two-component wave function in the electron-hole space, the quasiparticle en$\operatorname{ergy} E$ is measured with respect to the chemical potential $\mu$, and the hamiltonian $H_{0}$ within the superlattice period $a=d_{N}+d_{S}$, for $z \in\left(-d_{N}, d_{S}\right)$, is given by

$$
\begin{aligned}
H_{0}(\mathbf{r})=-\nabla \frac{\hbar^{2}}{2 m(\mathbf{r})} \nabla+\hat{W}[\delta(z)+\delta(z & \left.\left.+d_{N}\right)\right] \\
& +U(\mathbf{r})-\mu
\end{aligned}
$$

The first term is the quasiparticle kinetic energy in the effective mass approximation [1627, the second term, with $\hat{W}=\hbar^{2} k_{F S} Z / 2 m_{S}$, describes finite transparency of S-N interfaces modelled as $\delta$-function potential barriers, and dimensionless parameter $Z$ measures the barrier strength. Fermi energies in $\mathrm{N}$ and $\mathrm{S}$ layers are $E_{F N}=\hbar^{2} k_{F N}^{2} / 2 m_{N}=$ $\mu-U_{N}$ and $E_{F S}=\hbar^{2} k_{F S}^{2} / 2 m_{S}=\mu-U_{S}$, respectively. We define the corresponding effective chemical potentials as $\mu_{N}=\mu_{N}\left(k_{\|}\right)=E_{F N}-\left(\hbar^{2} k_{\|}^{2} / 2 m_{N}\right)$ and $\mu_{S}=\mu_{S}\left(k_{\|}\right)=$ $E_{F S}-\left(\hbar^{2} k_{\|}^{2} / 2 m_{S}\right)$, where $\mathbf{k}_{\|}$is the conserved quasiparticle momentum parallel to the layers.

The pair potential $\Delta(\mathbf{r})$ should be treated self-consistently. For the sake of simplicity we used the stepwise model with $\Delta(\mathbf{r})$ equal to constant $\Delta_{0}$ in $\mathrm{S}$ and zero in $\mathrm{N}$ layers 819. However, for $\mathrm{S} / \mathrm{N}$ superlattices with thin $\mathrm{S}$ layers, the effective $\Delta_{0}$ can be taken as the spaceaveraged self-consistently determined pair potential, correspondingly smaller than the bulk value 23 . For superlattices with thick S films, $\Delta_{0}$ can be set to the bulk value.

Solutions of BdG equation,

$$
\Psi(\mathbf{r})=\left(\begin{array}{c}
u(z) \\
v(z)
\end{array}\right) e^{i \mathbf{k}_{\|} \cdot \mathbf{r}},
$$

in $\mathrm{N}$ and $\mathrm{S}$ layers can be written in the form

$$
\begin{aligned}
\left(\begin{array}{l}
u(z) \\
v(z)
\end{array}\right)_{N}= & C_{1} \sin \left(k_{N}^{+} z\right)\left(\begin{array}{l}
1 \\
0
\end{array}\right)+C_{2} \cos \left(k_{N}^{+} z\right)\left(\begin{array}{l}
1 \\
0
\end{array}\right) \\
& +C_{3} \sin \left(k_{N}^{-} z\right)\left(\begin{array}{l}
0 \\
1
\end{array}\right)+C_{4} \cos \left(k_{N}^{-} z\right)\left(\begin{array}{l}
0 \\
1
\end{array}\right)
\end{aligned}
$$

and

$$
\begin{aligned}
\left(\begin{array}{c}
u(z) \\
v(z)
\end{array}\right)_{S} & =C_{5} \sin \left(k_{S}^{+} z\right)\left(\begin{array}{c}
\bar{u} \\
\bar{v}
\end{array}\right)+C_{6} \cos \left(k_{S}^{+} z\right)\left(\begin{array}{c}
\bar{u} \\
\bar{v}
\end{array}\right) \\
& +C_{7} \sin \left(k_{S}^{-} z\right)\left(\begin{array}{c}
\bar{v} \\
\bar{u}
\end{array}\right)+C_{8} \cos \left(k_{S}^{-} z\right)\left(\begin{array}{c}
\bar{v} \\
\bar{u}
\end{array}\right) .
\end{aligned}
$$

Here, $\Omega=\sqrt{E^{2}-\Delta_{0}^{2}}, k_{N}^{ \pm}=\sqrt{2 m_{N}\left(\mu_{N} \pm E\right) / \hbar^{2}}, k_{S}^{ \pm}=$ $\sqrt{2 m_{S}\left(\mu_{S} \pm \Omega\right) / \hbar^{2}}$ and the BCS coherence amplitudes are $\bar{u}=\sqrt{(1+\Omega / E) / 2}$ and $\bar{v}=\sqrt{(1-\Omega / E) / 2}$.

Complex coefficients $C_{1}$ through $C_{8}$ are determined from the boundary conditions at interfaces $z=0$ and $z=-d_{N}$ inside the primitive cell

$$
\left(\begin{array}{l}
u_{N}(0) \\
v_{N}(0)
\end{array}\right)=\left(\begin{array}{l}
u_{S}(0) \\
v_{S}(0)
\end{array}\right)
$$

$$
\begin{gathered}
\frac{1}{m_{N}}\left(\begin{array}{c}
u_{N}^{\prime}(0) \\
v_{N}^{\prime}(0)
\end{array}\right)+\frac{k_{F S}}{m_{S}} Z\left(\begin{array}{l}
u(0) \\
v(0)
\end{array}\right)=\frac{1}{m_{S}}\left(\begin{array}{l}
u_{S}^{\prime}(0) \\
v_{S}^{\prime}(0)
\end{array}\right), \\
e^{i K a}\left(\begin{array}{l}
u_{N}\left(-d_{N}\right) \\
v_{N}\left(-d_{N}\right)
\end{array}\right)=\left(\begin{array}{l}
u_{S}\left(d_{S}\right) \\
v_{S}\left(d_{S}\right)
\end{array}\right),
\end{gathered}
$$

$$
\begin{aligned}
\frac{e^{i K a}}{m_{N}}\left(\begin{array}{c}
u_{N}^{\prime}\left(-d_{N}\right) \\
v_{N}^{\prime}\left(-d_{N}\right)
\end{array}\right)-\frac{k_{F S}}{m_{S}} Z e^{i K a} & \left(\begin{array}{c}
u_{N}\left(-d_{N}\right) \\
v_{N}\left(-d_{N}\right)
\end{array}\right) \\
& =\frac{1}{m_{S}}\left(\begin{array}{c}
u_{S}^{\prime}\left(d_{S}\right) \\
v_{S}^{\prime}\left(d_{S}\right)
\end{array}\right) .
\end{aligned}
$$

Here, the Bloch condition $\Psi(x, y, z+a)=e^{i K a} \Psi(x, y, z)$ is used and the crystal momentum $K$ is taken within the first Brillouin zone, $K \in(-\pi / a, \pi / a)$.

Dispersion relation $E=E_{n, k_{\|}}(K)$ can be written in the following implicit form 8

$$
\begin{aligned}
\cos (K a) & =-\tilde{D}_{1} / 4 \pm \sqrt{\left(\tilde{D}_{1} / 4\right)^{2}-\tilde{D}_{2} / 4+1 / 2} \\
& \equiv F^{ \pm}\left(E, k_{\|}\right)
\end{aligned}
$$

where $\tilde{D}_{1}$ and $\tilde{D}_{2}$ are defined in terms of dimensionless quantities $E / \Delta_{0}, k_{\|} / k_{F S}, Z, d_{N} / \xi_{0}, d_{S} / \xi_{0}, m_{N} / m_{S}$, $E_{F N} / E_{F S}$, and $\Delta_{0} / E_{F S}$ (see the Appendix).

Global density of states (for both spin orientations) per unit area of the cross section $L_{x} L_{y}$, averaged over a primitive cell, is given by

$$
\begin{aligned}
g(E) & =\frac{1}{L_{x} L_{y}} \sum_{\sigma, k_{\|}, K} \delta\left(E-E\left(k_{\|}, K\right)\right) \\
& =\frac{1}{\pi} \int d k_{\|} k_{\|} \sum_{i=+,-} \frac{a}{2 \pi} \int d K^{i} \delta\left(E-E\left(k_{\|}, K^{i}\right)\right) \\
& =\frac{a}{2 \pi^{2}} \int d k_{\|} k_{\|} \sum_{i=+,-}\left|\frac{\partial K^{i}}{\partial E\left(k_{\|}, K^{i}\right)}\right|_{E\left(k_{\|}, K^{i}\right)=E},
\end{aligned}
$$

where $K^{ \pm}(E)$ are the solutions of Eq. (10), and

$$
\left|\frac{\partial K^{i}}{\partial E\left(k_{\|}, K^{i}\right)}\right|=\frac{1}{a}\left|\frac{\partial \arccos \left[F^{i}\left(E, k_{\|}\right)\right]}{\partial E}\right| .
$$

In accordance with Eq. (10), the integration over $k_{\|}$[or $\left.\mu_{S}=\mu_{S}\left(k_{\|}\right)\right]$in Eq. (11) is limited to the intervals given by

$$
\left(\tilde{D}_{1} / 4\right)^{2}-\tilde{D}_{2} / 4+1 / 2 \geq 0
$$

and

$$
\left|F^{ \pm}\left(E, k_{\|}\right)\right| \leq 1
$$

In the following, $g(E)$ is normalized to the normal-state value $\bar{g}=\left(m_{S} d_{S} k_{F S}+m_{N} d_{N} k_{F N}\right) / \pi^{2} \hbar^{2}$. 


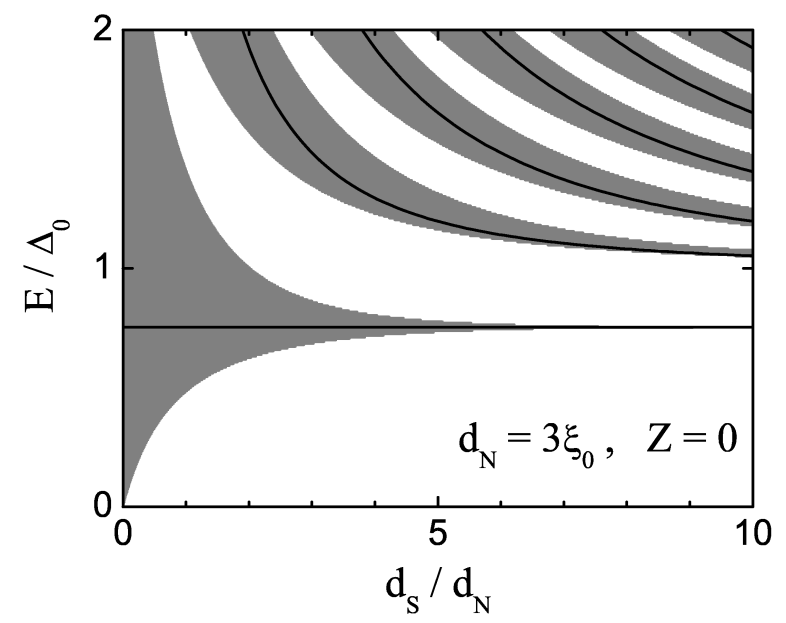

Fig. 1. Energy bands as a function of the $\mathrm{S}$ layer thickness $d_{S}$, for $\mathrm{S} / \mathrm{N}$ superlattices with thick $\mathrm{N}$ layers, $d_{N}=3 \xi_{0}$, transparent interfaces, $Z=0$, and $\mathbf{k}_{\|}=0$. Andreev bound states $\left(E<\Delta_{0}\right)$ and geometrical resonances $\left(E>\Delta_{0}\right)$ for the corresponding SNS and NSN trilayers are shown for comparison (solid curves).

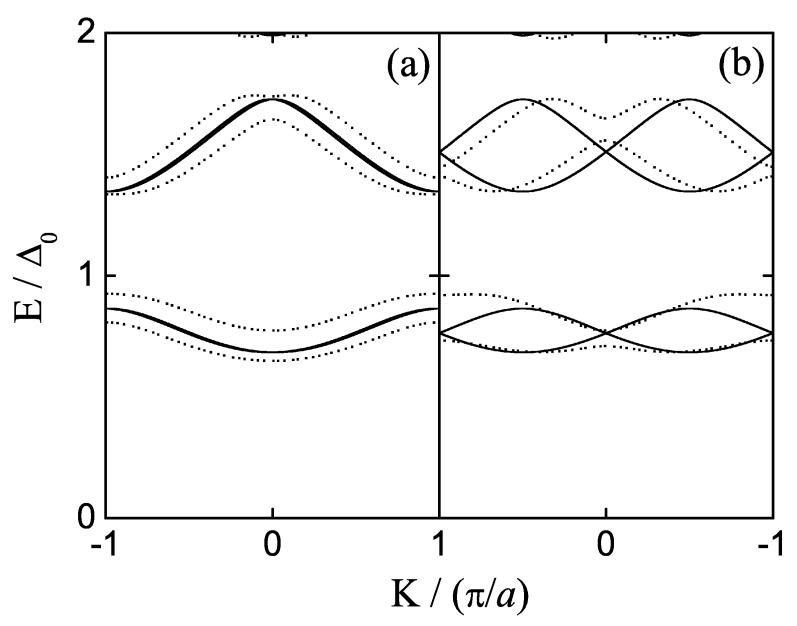

Fig. 2. Characteristic dispersion of energy bands illustrated for $d_{N}=3 \xi_{0}, Z=0, \mathbf{k}_{\|}=0$, and (a) $d_{S}=3.0005 d_{N}$, and (b) $d_{S}=3.0013 d_{N}$. Bands displayed in (a) are double degenerate for all $K$. Dotted curves represent dispersion for finite transparency $Z=0.5$.

\section{Energy bands and density of states}

The dispersion relation, Eq. (10), is solved numerically and the global density of states is calculated from Eq. (11) for various superlattices and for zero phase difference $\phi=$ 0 . In the following, we focus on the influence of finite interface transparency on quasiparticle band structure and density of states. For simplicity, this is illustrated for equal effective masses and Fermi wave-vectors, $m_{N} / m_{S}=1$ and $k_{F N} / k_{F S}=1$. Superconductors are characterized by the bulk value of the pair potential $\Delta_{0} / E_{F S}=10^{-3}$,

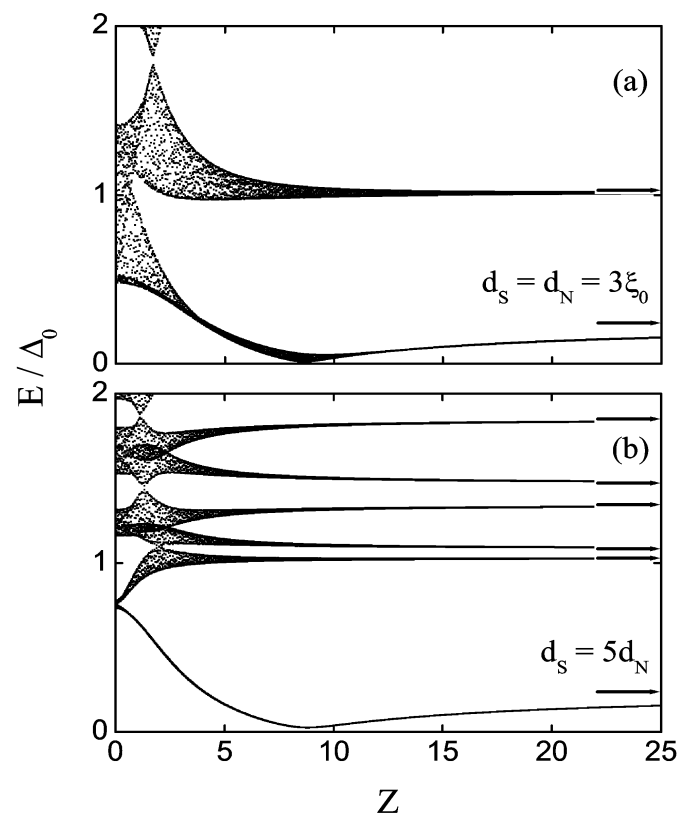

Fig. 3. Energy bands as a function of $Z$, for $\mathbf{k}_{\|}=0$ and for two particular S/N superlattices: (a) $d_{S}=d_{N}=3 \xi_{0}$ and (b) $d_{S}=15 \xi_{0}, d_{N}=3 \xi_{0}$. Arrows indicate the bound states in the tunnel limit.

which corresponds to the zero-temperature BCS coherence length $\xi_{0}=\hbar^{2} k_{F S} /\left(\pi m_{S} \Delta_{0}\right) \sim 10^{3} \AA$.

Energy bands for $\mathrm{S} / \mathrm{N}$ superlattices with thick $\mathrm{N}$ layers, transparent S-N interfaces and quasiparticles propagating perpendicular to the layers $\left(\mathbf{k}_{\|}=0\right)$ are shown in Fig. [1] Quasicontinuum of energy states corresponding to the crystal momentum within the first Brillouin zone, $K \in(-\pi / a, \pi / a)$, is indicated by shading the band width calculated from Eqs. (13) and (14).

For the corresponding SNS trilayer, Andreev bound states, $E<\Delta_{0}$, in the normal interlayer of thickness $d_{N}$, for zero phase difference across the junction, transparent interfaces, and $\mathbf{k}_{\|}=0$ are given by 28

$$
\frac{E_{n}}{\Delta_{0}}=\pi^{2}\left[n+\frac{1}{\pi} \arccos \left(\frac{E_{n}}{\Delta_{0}}\right)\right] \frac{1}{d_{N} / \xi_{0}}
$$

where $n=0,1, \ldots$ In this case the Andreev bound states are double degenerate. Geometrical resonances, $E>\Delta_{0}$, for the corresponding NSN junction with $\mathrm{S}$ interlayer of thickness $d_{S}$, and $Z=0, \mathbf{k}_{\|}=0$ are given by

$$
\frac{E_{n}}{\Delta_{0}}=\sqrt{1+n^{2} \frac{\pi^{4}}{\left(d_{S} / \xi_{0}\right)^{2}}},
$$

which follows from the condition $d_{S}\left(k_{S}^{+}-k_{S}^{-}\right)=2 n \pi$, where $n= \pm 1, \pm 2, \ldots$. At these energies the Andreev reflection vanishes and the electron is transmitted without creation or annihilation of Cooper pairs 222324. Both Andreev bound states and geometrical resonances of the corresponding SNS and NSN trilayers are shown in Fig. 1 for comparison. 
In $\mathrm{S} / \mathrm{N}$ superlattices with thick $\mathrm{S}$ layers, the energy band structure above the gap, $E>\Delta_{0}$, is also affected by the Andreev process 1718 . With increasing $d_{N}$, the band structure dependance on $d_{S} / d_{N}$ remains qualitatively the same as in Fig. 1] with compression and lowering of energy bands that enter the superconducting gap [29]. Andreev reflection is the fundamental mechanism that determines the quasiparticle band structure in $\mathrm{S} / \mathrm{N}$ superlattices. However, qualitatively the same results as shown in Fig. 1] are obtained for semiconductor / normal-metal superlattices 3031. Characteristic dispersion of energy bands, shown in Fig. 11 is illustrated in Fig. 2 for two close thicknesses of the $\mathrm{S}$ layer. For some layer thicknesses the energy bands are double degenerate for all $K$, Fig. 2 (a), in contrast with the usual degeneracy at high-symmetry points only (at the center and the ends of the first Brillouin zone), Fig. 2 (b). These two types of dispersion alternate rapidly with the change of layer thicknesses on the atomic scale $k_{F}^{-1}$, while the band width changes on the macroscopic scale.

Finite interface transparency, as well as mismatch of effective masses and Fermi wave-vectors 29, lift the degeneracy in $E(K)$, Fig. 2 and change the band structure, Fig. 3] For large $Z$, energy bands split into pairs of flat bands independent of $K$, and there is a significant change of the band energy below the superconducting gap. Approaching the tunnel limit for $Z \gg 1$, pairs of adjacent flat energy bands transform into bound states of isolated films defined by $d_{S} k_{S}^{ \pm}=n_{1} \pi$ and $d_{N} k_{N}^{ \pm}=n_{2} \pi$. However, this does not imply that the energy band splitting and decrease of the band widths due to the flattening will be visible in the $E$ vs. $d_{S(N)}$ plot (cf. Figs. 11 and 4). Energy levels for $Z \gg 1$ oscillate rapidly with layer thicknesses on the atomic scale $k_{F}^{-1}$, Fig. 5 so that $E$ vs. $d_{S(N)}$ curves fill the energy space quasicontinuously on the macroscopic scale, Fig. 4 This implies erasing of the band structure and localization of quasiparticle states in real superlattices with finite interface transparency and slightly unequal layers.

Previous analysis has been made for quasiparticles that propagate perpendicular to the layers. Dependence of energy bands on $k_{\|}$, i.e. on the effective chemical potential $\mu_{S}\left(k_{\|}\right)$, is illustrated for $Z=0$ in Figs. 6] (a) and [7 (a). Band widths decrease with the increase of $k_{\|}$, and bands split into pairs of bound states (flat bands) for very large parallel momentum [8, similar to the tunnel limit. The effect of erasing the band structure with finite interface transparency is enhanced with the increase of $k_{\|}$. Corresponding changes of the global density of states are shown in Figs. 6 (b) and 7 (b). Integration of Eq. (11) is performed over the shaded regions in Figs. 6] (a) and 7 (a), where Eqs. (13) and (14) are satisfied.

Now we shall focus on energy bands and the density of states in thin-layer $\mathrm{S} / \mathrm{N}$ superlattices, where coherence effects are pronounced and ballistic transport is more likely to take place 24]. Dependence of energy bands on the superlattice period is illustrated in Fig. 8 for $d_{S}=d_{N}$, $\mathbf{k}_{\|}=0$, and for both $Z=0$ and $Z=1$. It can be seen that the band structure in transparent thin-layer superlattices differs significantly from the thick-layer case considered

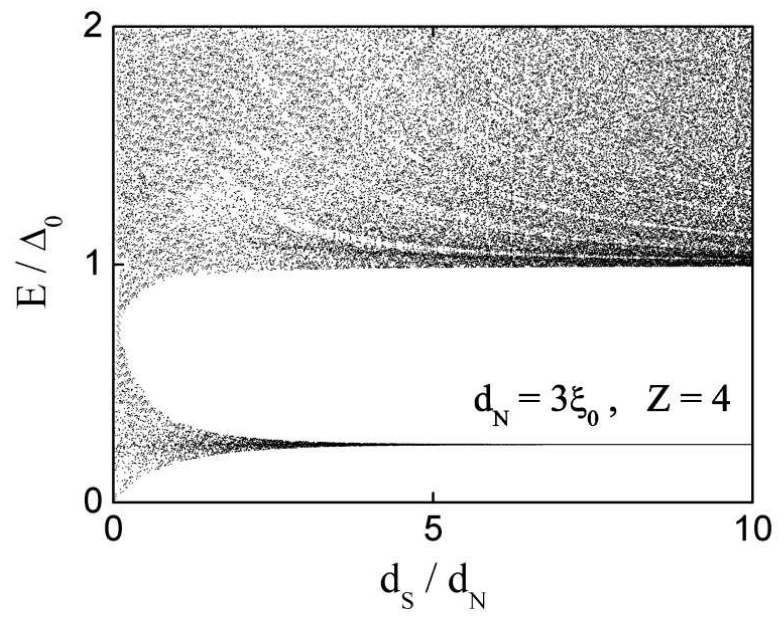

Fig. 4. Energy 'bands' for $d_{N}=3 \xi_{0}, \mathbf{k}_{\|}=0$ and $Z=4$. Shading is produced by the rapid oscillatory dependence of flat energy bands on the $\mathrm{S}$ layer thickness.

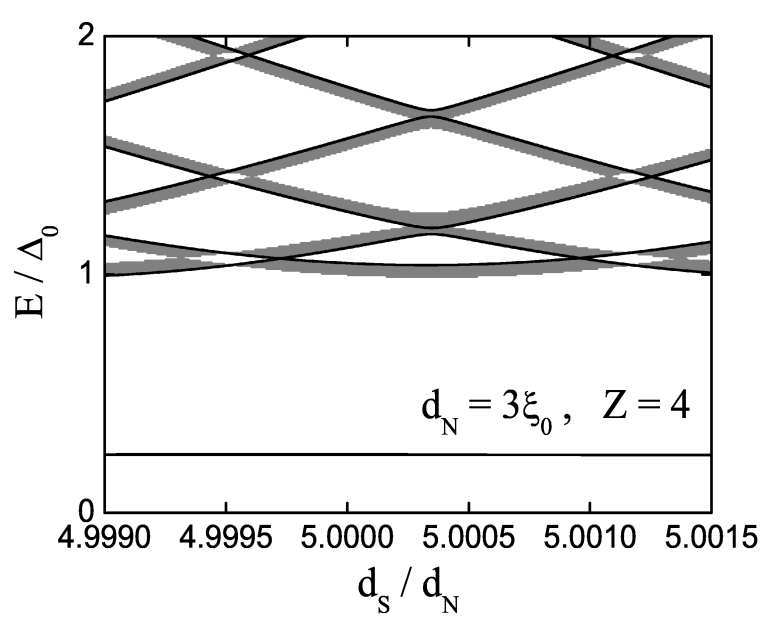

Fig. 5. Rapid oscillatory dependance of energy bands on the S layer thickness for nontransparent superlattices. Energy dispersion $E(K)$ is shown by shading for all $K$, solid curves represent $E(0)$.

in Ref. 8]. For thin layers, dispersion of energy bands is significant, with only a small part of the lower band laying below $\Delta_{0}$. Energy bands as a function of $k_{\|}$, and for various interface transparencies are shown in Fig. 9 For $Z=0$ and $d_{S}=d_{N}$, the most striking feature is the onset of the lowest energy band at the midgap, practically for any $k_{\|}$. This is not the case for thick-layer superlattices, where band energy decreases more rapidly down to zero with the increase of $k_{\|}$, resulting in the left-side "tail" of the subgap peak in the density of states, Figs. [6] and 7 For thin-layer superlattices, finite interface transparency introduces the resonance effect: energy bands penetrate periodically below the midgap with the increase of $k_{\|}$. This is more pronounced as $Z$ gets larger, Fig. 9] The cor- 


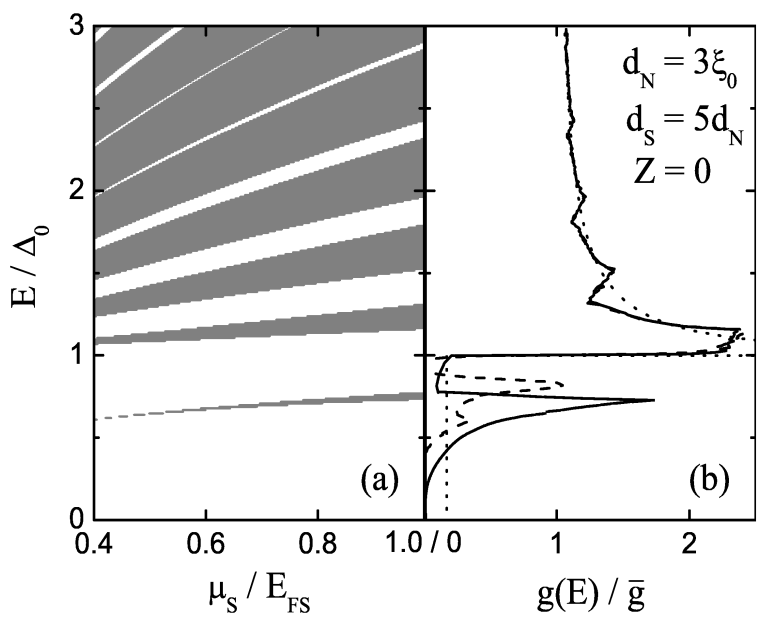

Fig. 6. (a) Normalized energy bands $E / \Delta_{0}$ as a function of $\mu_{S}=\mu_{S}\left(k_{\|}\right)$, for $\mathrm{S} / \mathrm{N}$ superlattice with $d_{S}=15 \xi_{0}, d_{N}=3 \xi_{0}$ and $Z=0$, and (b) the corresponding global density of states $g(E)$ normalized to the normal-state value $\bar{g}$. Global density of states for $Z=0.5$ (dashed curve), and in the tunnel limit (dotted curve) are given for comparison.

responding global densities of states for various interface transparencies are shown in Fig. 10

For transparent interfaces, the density of states is BCSlike with the energy gap $E_{g}$ smaller than the pair potential $\Delta_{0}$. The value of $E_{g}$ for transparent interfaces, equal effective masses, and equal Fermi energies can be obtained from the well known dispersion relation 6 6233

$$
\begin{aligned}
\cos \left[\left(K^{ \pm} \pm k_{z F}\right) a\right]= & \cos \left(q \delta d_{S}\right) \cos \left(q d_{N}\right) \\
& -\delta^{-1} \sin \left(q \delta d_{S}\right) \sin \left(q d_{N}\right),
\end{aligned}
$$

which is a special case of Eq. (10). Here, $k_{z F}=\sqrt{k_{F}^{2}-k_{\|}^{2}}$, $\delta=\Omega / E$, and $q=m E / \hbar^{2} k_{z F}$. For $d_{S}, d_{N} \rightarrow 0$, from Eqs. (11) and (17) exactly follows [1]

$$
E_{g}=\frac{\Delta_{0}}{1+d_{N} / d_{S}}
$$

Practically, this simple relation remains valid for the layer thicknesses up to one or two coherence lengths $\xi_{0}$, due to the weak variation of the bottom of the lowest energy band with the layer thickness, Fig. 8 (a).

With decreasing interfacial transparency, the subgap peak in $g(E)$ at $E_{g}$ decays, and the usual BCS coherence peak at $\Delta_{0}$ reenters as the superconducting layers become more isolated. In the tunnel limit, the BCS peak at $\Delta_{0}$ is completely restored, Fig. 10] (dotted curve in the bottom panel). For thicker layers $d_{S} \sim d_{N} \sim \xi_{0}$, the coherence effects are less pronounced and the tunnel limit behavior is practically reached for smaller $Z \sim 1$. Previously, this double peak structure in the density of states of $\mathrm{S} / \mathrm{N}$ superlattices is obtained within the tight binding approximation for atomic-scale layered systems, and apparently observed in high- $T_{c}$ intrinsically layered superconductors 4514.

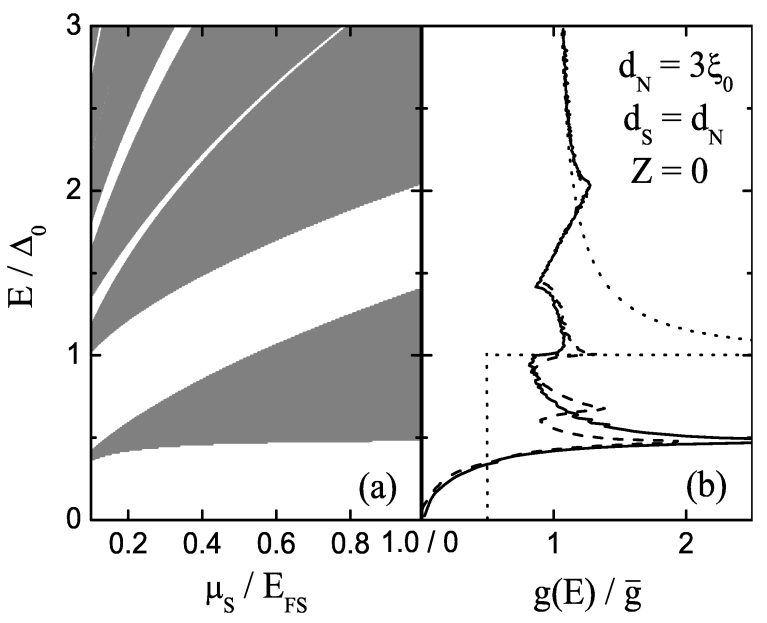

Fig. 7. (a) Normalized energy bands $E / \Delta_{0}$ as a function of $\mu_{S}=\mu_{S}\left(k_{\|}\right)$, for $\mathrm{S} / \mathrm{N}$ superlattice with $d_{S}=d_{N}=3 \xi_{0}$ and $Z=0$, and (b) the corresponding global density of states $g(E)$ normalized to the normal-state value $\bar{g}$. Global density of states for $Z=0.5$ (dashed curve), and in the tunnel limit (dotted curve) are given for comparison.

\section{Conclusion}

We have derived the dispersion relation for superconductor / normal-metal (semiconductor) superlattices in the clean limit, generalizing the previous expression of Plehn et al. 8. to include an arbitrary interface transparency and mismatch of effective band masses. The obtained general dispersion relation is used for numerical analysis of the

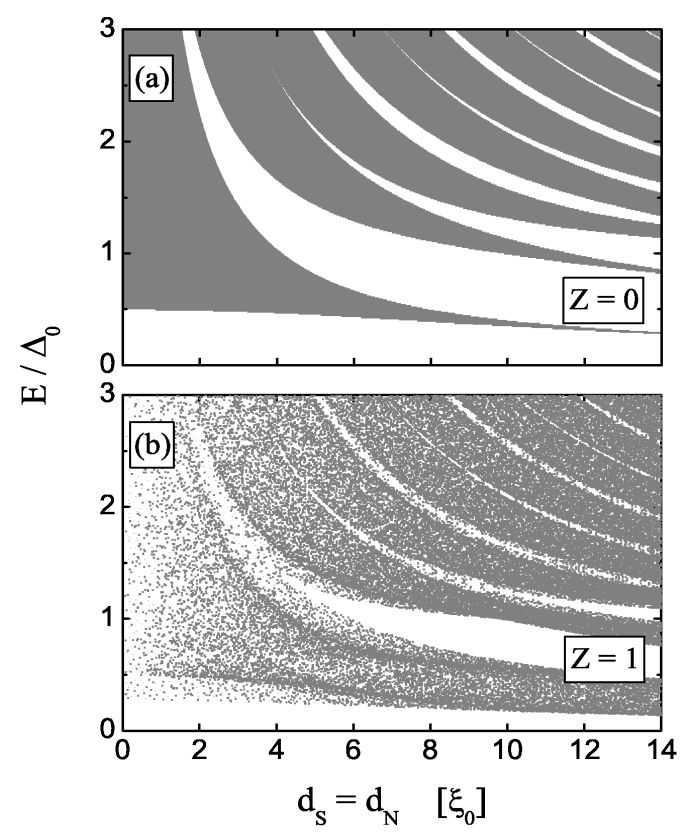

Fig. 8. (a) Energy bands for $\mathrm{S} / \mathrm{N}$ superlattices with $d_{S}=d_{N}$, $\mathbf{k}_{\|}=0$ and $Z=0$. (b) Erasing of the band structure with decrease of interface transparency is shown for $Z=1$. 


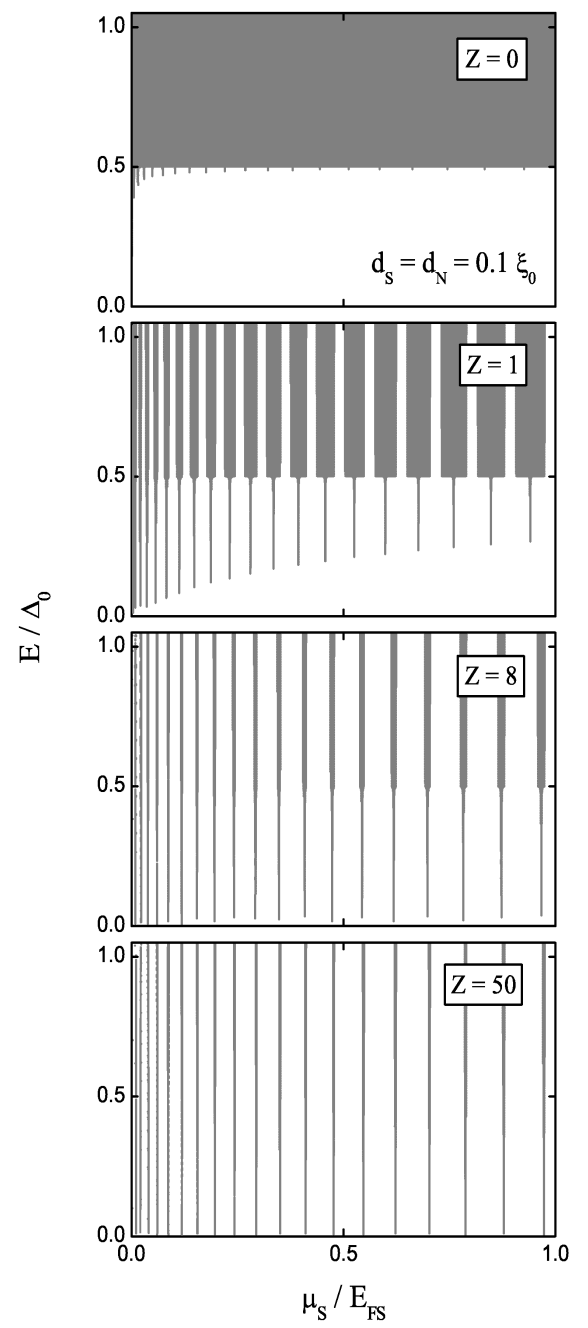

Fig. 9. Energy bands as a function of $\mu_{S}=\mu_{S}\left(k_{\|}\right)$, for $\mathrm{S} / \mathrm{N}$ superlattice with thin layers, $d_{S}=d_{N}=0.1 \xi_{0}$, and for various interface transparencies.

influence of interface transparency on energy band structure and density of states in metallic $\mathrm{S} / \mathrm{N}$ superlattices. Although we used stepwise approximation for the pair potential, our results will not be altered significantly by the fully self-consistent numerical calculations $[8$, if an effective pair potential (smaller than the bulk value) is taken for thin S layers, and simply the bulk value in the cases of thick S layers, low transparency, and mismatch of Fermi wave-vectors or band masses. Our results confirm previously obtained features in the metallic $\mathrm{S} / \mathrm{N}$ superlattices 678 , including the limiting cases of double barrier SNS or NSN trilayers 34182021222324, and are in a good qualitative agreement with the results obtained within the tight binding approximation [45].

Consequences of the quantum interference effect are strong and rapid (on the atomic scale) geometrical oscillations with layers thickness of the energy dispersion in transparent superlattices, and of the almost flat energy bands (transmission resonances) in the case of finite transparency. Oscillations in the latter case are practically

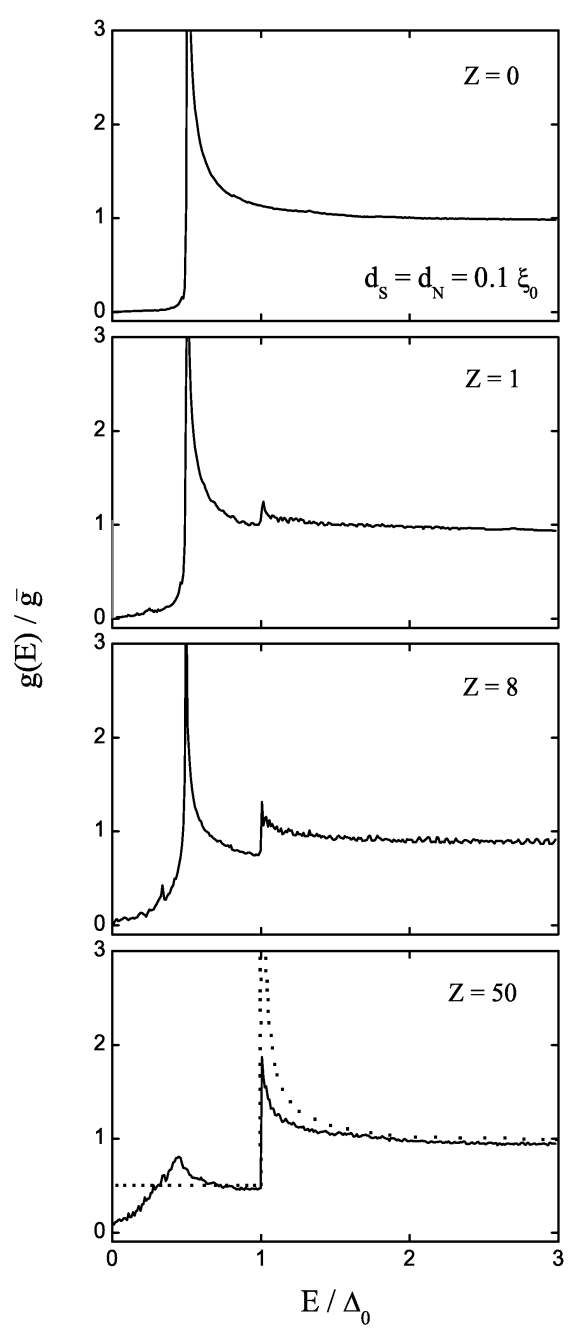

Fig. 10. Global density of states for $\mathrm{S} / \mathrm{N}$ superlattice with thin layers, $d_{S}=d_{N}=0.1 \xi_{0}$, and for various interface transparencies. Tunnel limit is indicated in the bottom panel (dotted curve). Note that the effective $\Delta_{0}$ varies with $Z$, being the smallest for $Z=0$ (top panel) and reaching the bulk value in the tunnel limit (bottom panel).

within the band width of the corresponding fully transparent superlattice. Practically, this could imply the localization of quasiparticle states in superlattices with low interface transparency.

Characteristic changes of quasiparticle band structure with decreasing interface transparency are suitably reflected in the global (averaged on the lattice period) density of states, which can be directly measured by STM techniques 1412. Oscillations of the density of states are simply related to the band structure for transparent superlattices with thick layers [6]. However, superlattices with the period smaller than the coherence length, when S and $\mathrm{N}$ layers lose their individual properties due to strong phase coupling by Andreev scattering, do not differ significantly from a bulk BCS superconductor, except for a subgap peak in the global density of states instead of the superconductor coherence peak at $\Delta_{0}$ [4]. For transpar- 
ent interfaces, position of the subgap peak is simply related to the lattice parameters. For finite interface transparency, we find the characteristic double peak structure in the global density of states [5]. With decreasing transparency the subgap peak decreases, only slightly changing the position, while the coherence peak at the effective $\Delta_{0}$ grows, and the density of states develops towards the gapless BCS result for the bulk superconductor in the tunnel limit. We point out that this double-peak structure of the global density of states in small-period clean-metal S/N superlattices can be used as a reliable experimental probe for interface transparency.

We are grateful to Ivana Petković, Miloš Božović, and Boris Grbić for useful discussions. The work has been supported by the Serbian Ministry of Science, Project No. 1899.

\section{References}

1. M.V. Baranov, A.I. Buzdin, L.N. Bulaevskii, Zh. Eksp. Teor. Fiz. 91, 1063 (1986) [Sov. Phys. JETP 64, 628 (1986)] 2. S. Takahashi, M. Tachiki, Phys. Rev. B 33, 4620 (1986); S. Takahashi, M. Tachiki, Phys. Rev. B 34, 3162 (1986)

3. Z. Radović, M. Ledvij, L. Dobrosavljević-Grujić, Phys. Rev. B 43, 8613 (1991)

4. L.N. Bulaevskii, M.V. Zyskin, Phys. Rev. B 42, 10230 (1990)

5. A.I. Buzdin, V.P. Damjanović, A.Yu. Simonov, Physica C 194, 109 (1992)

6. Yu. Tanaka, M. Tsukada, Phys. Rev. B 44, 7578 (1991)

7. B.P. Stojković, O.T. Valls, Phys. Rev. B 50, 3374 (1994)

8. H. Plehn, O.-J. Wacker, R. Kümmel, Phys. Rev. B 49, 12140 (1994)

9. S.V. Kuplevakhsky, S.V. Naydenov, A.A. Galiautdinov, Phys. Rev. B 56, 7858 (1997); S.V. Kuplevakhsky, A.V. Naduev, S.V. Naydenov, Superlatt. Microstruct. 25, 819 (1999)

10. R. Koperdraad, Ph.D. thesis, Vrije Universiteit Amsterdam, 1995

11. J.B. Ketterson, S.N. Song, Superconductivity (Cambridge University Press, 1999)

12. N. Moussy, H. Curtois, B. Pannetier, Europhys. Lett. 55, 861 (2001); N. Moussy, H. Courtois, B. Pannetier, Rev. Sci. Instrum. 72, 128 (2001)

13. B.-L. Huang, C.-Yu Mou, Physica C 390, 167 (2003)

14. L. Buschmann, M. Boekholt, G. Güntherodt, Physica C 203, 68 (1992)

15. S. Kashiwaya et al., Phys. Rev. B 53, 2667 (1996)

16. N.A. Mortensen, K. Flensberg, A.-P. Jauho, Phys. Rev. B 59, 10176 (1999)

17. O. Šipr, B. Györffy, J. Phys. C 8, 169 (1996)

18. U. Gunsenheimer, U. Schüssler, R. Kümmel, Phys. Rev. B 49, 6111 (1994)

19. Yu. Tanaka, M. Tsukada, Phys. Rev. B 47, 287 (1993)

20. A. Brinkman, A.A. Golubov, Phys. Rev. B 61, 11297 (2000)

21. Z. Radović, N. Lazarides, N. Flytzanis, Phys. Rev. B 68, 014501 (2003)

22. M. Božović, Z. Radović, Phys. Rev. B 66, 134524 (2002)

23. M. Božović, Z. Radović, Europhys. Lett. 70, 513 (2005)
24. M. Božović, Z. Pajović, Z. Radović, Physica C 391, 309 (2003)

25. A.F. Andreev, Pis'ma Zh. Eksp. Teor. Fiz. 46, 1823 (1964) [JETP Lett. 19, 1228 (1964)]

26. K. Halterman, O.T. Valls, Phys. Rev. B 66, 224516 (2002)

27. R.A. Morrow, K.R. Brownstein, Phys. Rev. B 30, 678 (1984)

28. I.O. Kulik, Zh. Eksp. Teor. Fiz. 57, 1745 (1969) [Sov. Phys. JETP 30, 944 (1970)]

29. M. Vanević, Diploma thesis, University of Belgrade, 2003

30. M. Steslicka et al., Surf. Sci. Rep. 47, 93 (2002)

31. E.L. Ivchenko, G.E. Pikus, Superlattices and Other Heterostructures - Symmetry and Optical Phenomena, 2nd edn. (Springer, Berlin, 1997)

32. A.P. van Gelder, Phys. Rev. 181, 787 (1969)

33. R. Kümmel, Phys. Rev. B 3, 784 (1971)

34. U. Schüssler, R. Kümmel, Phys. Rev. B 47, 2754 (1993) 


\section{Appendix}

From the boundary conditions, Eqs. (61)-(9), the dispersion relation, Eq. (10), is expressed through $\tilde{D}_{1}=D_{1} / D_{0}$ and $\tilde{D}_{2}=D_{2} / D_{0}$, where

$$
\begin{aligned}
& D_{0}=m_{r}^{2} k_{N}^{-} k_{N}^{+} k_{S}^{-} k_{S}^{+}\left(\bar{u}^{2}-\bar{v}^{2}\right)^{2}, \\
& D_{1}=F_{0}+F_{1}\left(Z k_{F S}\right)+F_{2}\left(Z k_{F S}\right)^{2}, \\
& D_{2}=G_{0}+G_{1}\left(Z k_{F S}\right)+G_{2}\left(Z k_{F S}\right)^{2}+G_{3}\left(Z k_{F S}\right)^{3}+G_{4}\left(Z k_{F S}\right)^{4} .
\end{aligned}
$$

Here, $m_{r}=m_{N} / m_{S}, F_{0}$ through $F_{2}$ and $G_{0}$ through $G_{4}$ are given by

$$
\begin{gathered}
F_{0}=m_{r}\left(\bar{u}^{2}-\bar{v}^{2}\right)\{[ \\
{\left[k_{N}^{+} k_{S}^{+} s_{N}^{-} s_{S}^{-}\left(k_{N}^{-2}+m_{r}^{2} k_{S}^{-2}\right)+k_{N}^{-} k_{S}^{-} s_{N}^{+} s_{S}^{+}\left(k_{N}^{+2}+m_{r}^{2} k_{S}^{+2}\right)\right] \bar{u}^{2}} \\
-\left[k_{N}^{-} k_{S}^{+} s_{N}^{+} s_{S}^{-}\left(k_{N}^{+2}+m_{r}^{2} k_{S}^{-2}\right)+k_{N}^{+} k_{S}^{-} s_{N}^{-} s_{S}^{+}\left(k_{N}^{-2}+m_{r}^{2} k_{S}^{+2}\right)\right] \bar{v}^{2} \\
\left.+2 m_{r} k_{N}^{-} k_{N}^{+} k_{S}^{-} k_{S}^{+}\left[\bar{v}^{2}\left(c_{N}^{+} c_{S}^{-}+c_{N}^{-} c_{S}^{+}\right)-\bar{u}^{2}\left(c_{N}^{+} c_{S}^{+}+c_{N}^{-} c_{S}^{-}\right)\right]\right\}, \\
F_{1}=-2 m_{r}^{2}\left(\bar{u}^{2}-\bar{v}^{2}\right)\left\{k_{N}^{-} k_{N}^{+}\left[k_{S}^{+} s_{S}^{-}\left(c_{N}^{-} \bar{u}^{2}-c_{N}^{+} \bar{v}^{2}\right)+k_{S}^{-} s_{S}^{+}\left(c_{N}^{+} \bar{u}^{2}-c_{N}^{-} \bar{v}^{2}\right)\right]\right. \\
\left.+m_{r} k_{S}^{-} k_{S}^{+}\left[k_{N}^{-} s_{N}^{+}\left(c_{S}^{+} \bar{u}^{2}-c_{S}^{-} \bar{v}^{2}\right)+k_{N}^{+} s_{N}^{-}\left(c_{S}^{-} \bar{u}^{2}-c_{S}^{+} \bar{v}^{2}\right)\right]\right\}, \\
F_{2}=-m_{r}^{3}\left(\bar{u}^{2}-\bar{v}^{2}\right)\left[k_{N}^{-} s_{N}^{+}\left(k_{S}^{-} s_{S}^{+} \bar{u}^{2}-k_{S}^{+} s_{S}^{-} \bar{v}^{2}\right)+k_{N}^{+} s_{N}^{-}\left(k_{S}^{+} s_{S}^{-} \bar{u}^{2}-k_{S}^{-} s_{S}^{+} \bar{v}^{2}\right)\right],
\end{gathered}
$$

and

$$
\begin{aligned}
G_{0}=s_{N}^{-} s_{N}^{+} s_{S}^{-} s_{S}^{+}\left(k_{N}^{-2} k_{N}^{+2}+m_{r}^{4} k_{S}^{-2} k_{S}^{+2}\right)\left(\bar{u}^{2}-\bar{v}^{2}\right)^{2} & \\
-2\left(\bar{u}^{2}-\bar{v}^{2}\right)\{ & m_{r} k_{N}^{-} k_{N}^{+}\left[k_{N}^{+} c_{N}^{-} s_{N}^{+}\left(k_{S}^{-} c_{S}^{-} s_{S}^{+} \bar{u}^{2}-k_{S}^{+} c_{S}^{+} s_{S}^{-} \bar{v}^{2}\right)+k_{N}^{-} c_{N}^{+} s_{N}^{-}\left(k_{S}^{+} c_{S}^{+} s_{S}^{-} \bar{u}^{2}-k_{S}^{-} c_{S}^{-} s_{S}^{+} \bar{v}^{2}\right)\right] \\
& \left.+m_{r}^{3} k_{S}^{-} k_{S}^{+}\left[k_{N}^{-} c_{N}^{-} s_{N}^{+}\left(k_{S}^{+} c_{S}^{-} s_{S}^{+} \bar{u}^{2}-k_{S}^{-} c_{S}^{+} s_{S}^{-} \bar{v}^{2}\right)+k_{N}^{+} c_{N}^{+} s_{N}^{-}\left(k_{S}^{-} c_{S}^{+} s_{S}^{-} \bar{u}^{2}-k_{S}^{+} c_{S}^{-} s_{S}^{+} \bar{v}^{2}\right)\right]\right\} \\
+m_{r}^{2}\left(2 k_{N}^{-} k_{N}^{+}\{\right. & s_{S}^{-} s_{S}^{+} \bar{u}^{2} \bar{v}^{2}\left(c_{N}^{-} c_{N}^{+}-1\right)\left(k_{S}^{-2}+k_{S}^{+2}\right) \\
& \left.+k_{S}^{-} k_{S}^{+}\left[\left(1+2 c_{N}^{-} c_{N}^{+} c_{S}^{-} c_{S}^{+}\right)\left(\bar{u}^{4}+\bar{v}^{4}\right)-2 \bar{u}^{2} \bar{v}^{2}\left(c_{S}^{-} c_{S}^{+}+c_{N}^{-} c_{N}^{+}+c_{N}^{-} c_{N}^{+} c_{S}^{-} c_{S}^{+}\right)\right]\right\} \\
+s_{N}^{-} s_{N}^{+} & \left\{2 k_{S}^{-} k_{S}^{+} \bar{u}^{2} \bar{v}^{2}\left(c_{S}^{-} c_{S}^{+}-1\right)\left(k_{N}^{-2}+k_{N}^{+2}\right)\right. \\
& \left.\left.+s_{S}^{-} s_{S}^{+}\left[\bar{u}^{4}\left(k_{N}^{-2} k_{S}^{+2}+k_{N}^{+2} k_{S}^{-2}\right)+\bar{v}^{4}\left(k_{N}^{-2} k_{S}^{-2}+k_{N}^{+2} k_{S}^{+2}\right)\right]\right\}\right)
\end{aligned}
$$

$$
\begin{aligned}
& G_{1}=-2 m_{r}\left(\bar{u}^{2}-\bar{v}^{2}\right) \\
& \times\left[s _ { N } ^ { + } \left(m _ { r } s _ { N } ^ { - } \left\{c_{S}^{+} k_{S}^{+} s_{S}^{-}\left[k_{N}^{-2} \bar{u}^{2}-k_{N}^{+2} \bar{v}^{2}+m_{r}^{2} k_{S}^{-2}\left(\bar{u}^{2}-\bar{v}^{2}\right)\right]\right.\right.\right. \\
& \left.+c_{S}^{-} k_{S}^{-} s_{S}^{+}\left[k_{N}^{+2} \bar{u}^{2}-k_{N}^{-2} \bar{v}^{2}+m_{r}^{2} k_{S}^{+2}\left(\bar{u}^{2}-\bar{v}^{2}\right)\right]\right\} \\
& \left.+c_{N}^{-} k_{N}^{-}\left\{-2 c_{S}^{-} c_{S}^{+} m_{r}^{2} k_{S}^{-} k_{S}^{+}\left(\bar{u}^{2}-\bar{v}^{2}\right)+s_{S}^{-} s_{S}^{+}\left[k_{N}^{+2}\left(\bar{u}^{2}-\bar{v}^{2}\right)+m_{r}^{2}\left(k_{S}^{+2} \bar{u}^{2}-k_{S}^{-2} \bar{v}^{2}\right)\right]\right\}\right) \\
& +c_{N}^{+} k_{N}^{+}\left(-2 c_{S}^{-} m_{r} k_{S}^{-}\left(c_{S}^{+} m_{r} s_{N}^{-} k_{S}^{+}+c_{N}^{-} k_{N}^{-} s_{S}^{+}\right)\left(\bar{u}^{2}-\bar{v}^{2}\right)\right. \\
& \left.\left.+s_{S}^{-}\left\{-2 c_{N}^{-} c_{S}^{+} m_{r} k_{N}^{-} k_{S}^{+}\left(\bar{u}^{2}-\bar{v}^{2}\right)+s_{N}^{-} s_{S}^{+}\left[k_{N}^{-2}\left(\bar{u}^{2}-\bar{v}^{2}\right)+m_{r}^{2}\left(k_{S}^{-2} \bar{u}^{2}-k_{S}^{+2} \bar{v}^{2}\right)\right]\right\}\right)\right], \\
& G_{2}=-m_{r}^{2}\left(\bar{u}^{2}-\bar{v}^{2}\right) \\
& \times\left(2 c_{S}^{-} m_{r} k_{S}^{-}\left\{2 c_{S}^{+} m_{r} s_{N}^{-} s_{N}^{+} k_{S}^{+}\left(-\bar{u}^{2}+\bar{v}^{2}\right)+s_{S}^{+}\left[-c_{N}^{-} k_{N}^{-} s_{N}^{+}\left(\bar{u}^{2}-2 \bar{v}^{2}\right)+c_{N}^{+} k_{N}^{+} s_{N}^{-}\left(-2 \bar{u}^{2}+\bar{v}^{2}\right)\right]\right\}\right. \\
& +s_{S}^{-}\left(-2 c_{N}^{+} k_{N}^{+}\left[c_{S}^{+} m_{r} s_{N}^{-} k_{S}^{+}\left(\bar{u}^{2}-2 \bar{v}^{2}\right)+2 c_{N}^{-} k_{N}^{-} s_{S}^{+}\left(\bar{u}^{2}-\bar{v}^{2}\right)\right]\right. \\
& \left.\left.+s_{N}^{+}\left\{s_{N}^{-}\left[k_{N}^{-2}+k_{N}^{+2}+m_{r}^{2}\left(k_{S}^{-2}+k_{S}^{+2}\right)\right] s_{S}^{+}\left(\bar{u}^{2}-\bar{v}^{2}\right)+2 c_{N}^{-} c_{S}^{+} m_{r} k_{N}^{-} k_{S}^{+}\left(-2 \bar{u}^{2}+\bar{v}^{2}\right)\right\}\right)\right), \\
& G_{3}=2 m_{r}^{3}\left(\bar{u}^{2}-\bar{v}^{2}\right)^{2}\left\{m_{r} k_{S}^{+} s_{N}^{-} s_{N}^{+} s_{S}^{-} c_{S}^{+}+s_{S}^{+}\left[k_{N}^{-} c_{N}^{-} s_{N}^{+} s_{S}^{-}+s_{N}^{-}\left(m_{r} k_{S}^{-} c_{S}^{-} s_{N}^{+}+k_{N}^{+} c_{N}^{+} s_{S}^{-}\right)\right]\right\},
\end{aligned}
$$




$$
G_{4}=m_{r}^{4} s_{N}^{-} s_{N}^{+} s_{S}^{-} s_{S}^{+}\left(\bar{u}^{2}-\bar{v}^{2}\right)^{2},
$$

where $s_{N}^{ \pm} \equiv \sin \left(k_{N}^{ \pm} d_{N}\right), c_{N}^{ \pm} \equiv \cos \left(k_{N}^{ \pm} d_{N}\right), s_{S}^{ \pm} \equiv \sin \left(k_{S}^{ \pm} d_{S}\right)$, and $c_{S}^{ \pm} \equiv \cos \left(k_{S}^{ \pm} d_{S}\right)$. For $Z=0$ and $m_{r}=1$ expressions for $\tilde{D}_{1}$ and $\tilde{D}_{2}$ reduce to the results given in Ref. [8] . 